\title{
ANALYTIC DOMINATION BY FRACTIONAL POWERS OF A POSITIVE OPERATOR
}

\author{
BY ROE GOODMAN ${ }^{1}$ \\ Communicated by Gian-Carlo Rota, March 11, 1968
}

Introduction. Let $A$ be an (unbounded) linear operator on a Banach space $\mathfrak{S}$. An analytic vector for $A$ is an element $u \in \mathfrak{S}$ such that $A^{n} u$ is defined for all $n$ and

$$
\sum_{n=0}^{\infty} \frac{\left\|A^{n} u\right\|}{n !} t^{n}<\infty
$$

for some $t>0$, i.e. the power series expansion of $e^{t A} u$ is defined and has a positive radius of absolute convergence.

Nelson [2] introduced and studied the notion of analytic domination of one operator (or a family of operators) by another: $A$ analytically dominates the operator $X$ if every analytic vector for $A$ is an analytic vector for $X$. In $\$ 1$ we announce an analytic domination theorem; the hypotheses were suggested by Nelson's treatment of Lie algebras of skew-symmetric operators in [2], while the conclusion was suggested by some results of Kotake and Narasimhan [1]. We apply our theorem in $\$ 2$ to the characterization of analytic vectors for a unitary representation of a Lie group.

1. Analytic domination. Let $\mathfrak{S}$ be a complex Hilbert space, and $A$ a positive, selfadjoint operator on $\mathfrak{S}$, which we normalize so that $A \geqq I$. If $\alpha$ is a complex number, the operator $A^{\alpha}$ is defined via the operational calculus for selfadjoint operators, and $\mathfrak{D}\left(A^{\alpha}\right) \subseteq \mathfrak{D}\left(A^{\beta}\right)$ if $\operatorname{Re} \alpha \geqq \operatorname{Re} \beta$. (For any operator $T$ on $\mathfrak{S}, \mathfrak{D}(T)$ will denote its domain of definition.) Let

$$
\mathfrak{S}^{\infty}=\bigcap_{n=1}^{\infty} \mathfrak{D}\left(A^{n}\right)
$$

(the $C^{\infty}$-vectors for $A$ ). Then we have the following analytic domination criterion: $(\operatorname{ad} X(A)=X A-A X)$.

TheOREM 1. Let $X: \mathfrak{S}^{\infty} \rightarrow \mathfrak{S}^{\infty}$ be symmetric or skew-symmetric. Suppose that for some $\alpha, 0<\alpha<1$,

$$
\|X u\| \leqq\left\|A^{\alpha} u\right\|,
$$

${ }^{1}$ This research was supported in part by Air Force OSR Contract \#F 44620-67. C-0008. 
(2)

$$
\left\|(\operatorname{ad} X)^{n}(A) u\right\| \leqq n !\|A u\|
$$

for all $u \in \mathfrak{S}^{\infty}$. Then every analytic vector for $A^{\alpha}$ is an analytic vector for $X$.

The proof of Theorem 1 shows slightly more, namely

CoROLlaRy 1.1. Suppose $u \in \mathfrak{S}^{\infty}$ and $\left\|A^{n} u\right\| \leqq M^{n} n^{n / \alpha}$, for some constant $M$. Then $u$ is an analytic vector for $X$, and there exists a constant $C$ depending only on $M$ and $\alpha$ such that $\left\|X^{n} u\right\| \leqq C^{n} n !$.

If we eliminate the assumption of symmetry or skew-symmetry on $X$, then the proof of Theorem 1 yields (we use the notation $(u \mid v)$ for the inner product in $\mathfrak{S}$ ):

Corollary 1.2. Suppose $X: \mathfrak{S}^{\infty} \rightarrow \mathfrak{S g}^{\infty}$ and $X$ has an adjoint

$$
X^{+}: \mathfrak{S}^{\infty} \rightarrow \mathfrak{S}^{\infty}
$$

(i.e. $(X u \mid v)=\left(u \mid X^{+} v\right)$ for $\left.u, v \in \mathfrak{S}^{\infty}\right)$. Suppose conditions (1) and (2) of Theorem 1 are satisfied by both $X$ and $X^{+}$. Then the conclusions of Theorem 1 and Corollary 1.1 hold for $X$ (and for $\left.X^{+}\right)$.

Remarks. The case $\alpha=0$ of the theorem is trivial, since it implies $X$ bounded. The case $\alpha=1$ is Nelson's analytic domination theorem, [2, Corollary 3.2]. Our proof, roughly speaking, proceeds by first showing that one may replace $A$ by $A^{\alpha}$ in (2), and then applying Nelson's theorem relative to $A^{\alpha}$ and $X$.

The idea of the proof is quite simple: we observe that $A^{\alpha}$ can be expressed in terms of an integral involving $A(A+\lambda)^{-1}, \lambda \geqq 0$; hence we can estimate $(\operatorname{ad} X)^{n}\left(A^{\alpha}\right)$ in terms of $(\operatorname{ad} X)^{n}\left[A(A+\lambda)^{-1}\right]$. The precise inequalities, however, are somewhat subtle. Direct norm estimates lead to a logarithmically divergent integral; we must use the symmetry of $X$ and $A$ together with interpolation on suitable fractional quadratic norms in order to obtain the needed a priori estimates for Nelson's theorem.

2. Analytic vectors for unitary representations. Let $G$ be a Lie group, (5) its Lie algebra, and suppose $U$ is a continuous unitary representation of $G$ on a Hilbert space $\mathfrak{S}$. To every vector $v \in \mathfrak{S}$ we associate its trajectory $\tilde{v}$ under $U$. We say that $v$ is a $C^{\infty}$ (resp. analytic) vector if $\tilde{v}$ is infinitely differentiable (resp. real analytic) as an $\mathfrak{S}$-valued function on $G$, and we denote the corresponding subspaces of $\mathfrak{S}$ by $\mathfrak{S}^{\infty}$ and $\mathfrak{S}^{\omega}$. On $\mathfrak{S}^{\infty}, U$ defines a representation of $\mathbb{S}$ by skewsymmetric operators. (See [2].) 
Let $X_{1}, \cdots, X_{d}$ be a basis for $\mathbb{G}$, and set $\Delta=\sum_{k=1}^{d} X_{k}^{2}$. The operator $U(1-\Delta)$ is symmetric on $\mathfrak{S}^{\infty}$ and its closure, which we denote by $A$, is a positive selfadjoint operator, $A \geqq 1$ [2]. Furthermore the space $\mathfrak{S}^{\infty}$ of infinitely differentiable vectors for the representation is definable in terms of $A$, namely

$$
\mathfrak{S}^{\infty}=\bigcap_{n=1}^{\infty} \mathfrak{D}\left(A^{n}\right)
$$

([2, Corollary 9.3]). Nelson also proved that every analytic vector for $A$ was in $\mathfrak{S}^{\omega}$, by employing his analytic domination theorem. By using our Theorem 1 , we can obtain a sharper result. Set $B=A^{1 / 2}$. Then we have

THEOREM 2. $\mathfrak{S E}^{\omega}$ is precisely the set of analytic vectors for $B$.

Using the more explicit estimates of Corollary 1.1, we obtain

Corollary 2.1 Let $v \in \mathfrak{S}^{\infty}$. Then $v \in \mathfrak{S}^{\omega}$ if and only if there exists a constant $M$ such that

$$
\left\|U(\Delta)^{n} v\right\| \leqq M^{n}(2 n) !
$$

for all $n$. In this case there exists a neighborhood $V$ of 0 in (\$) depending only on $M$ such that

$$
\sum_{n=0}^{\infty} \frac{1}{n !} U(X)^{n} v
$$

is absolutely convergent for $X \in V$.

\section{REFERENCES}

1. T. Kotake and M. S. Narasimhan, Regularity theorems for fractional powers of a linear operator, Bull. Soc. Math. France 90 (1962), 449-471.

2. E. Nelson, Analytic vectors, Ann. of Math. 70 (1959), 572-615.

Massachusetts Institute of Technology 